\title{
Strong Measurements Give a Better Direct Measurement of the Quantum Wave Function
}

\author{
Giuseppe Vallone and Daniele Dequal \\ Department of Information Engineering, University of Padova, via Gradenigo 6/B, 35131 Padova, Italy \\ (Received 24 April 2015; revised manuscript received 24 December 2015; published 29 January 2016)

\begin{abstract}
Weak measurements have thus far been considered instrumental in the so-called direct measurement of
\end{abstract} \\ the quantum wave function [J. S. Lundeen, Nature (London) 474, 188 (2011).]. Here we show that a direct \\ measurement of the wave function can be obtained by using measurements of arbitrary strength. In \\ particular, in the case of strong measurements, i.e., those in which the coupling between the system and the \\ measuring apparatus is maximum, we compared the precision and the accuracy of the two methods, by \\ showing that strong measurements outperform weak measurements in both for arbitrary quantum states \\ in most cases. We also give the exact expression of the difference between the original and reconstructed \\ wave function obtained by the weak measurement approach; this will allow one to define the range of \\ applicability of such a method.
}

DOI: 10.1103/PhysRevLett.116.040502

In quantum mechanics, the wave function is the fundamental representation of any quantum system, and it offers the key tool for predicting the measurement outcomes of a physical apparatus. Its determination is therefore of crucial importance in many applications. In order to reconstruct the complete quantum wave function of a system, an indirect method, know as quantum state tomography (QST), has been developed [1]. QST is based on the measurement of complementary variables of several copies of the same quantum system, followed on an estimation of the wave function that better reproduce the results obtained. This method, originally proposed for a two-level system, has been extended to a generic number of discrete quantum states [2] as well as to a continuous variable state [3]. Recently, Lundeen et al. [4] proposed an alternative operational definition of the wave function based on the weak measurement [5-7]. After the first demonstration, in which the transverse wave function of a photon has been measured, this method has been applied for the measurement of the photon polarization [8], its angular momentum [9], and its trajectory [10]. The method has been subsequently generalized to mixed states [11] to continuous variable systems [12] and compared to standard quantum state tomography in Refs. [13,14].

By such a method, that we call direct weak tomography (DWT), a "direct measurement" of the quantum wave function is obtained: the term direct measurement refers to the property that a value proportional to the wave function appears straight from the measured probabilities without further complicated calculations or fitting on the measurement outcomes [15]. As originally proposed [4], the method is based on the weak measurement obtained by a "weak" interaction between the "pointer" (i.e., the measurement apparatus) and the system. Weak measurements occur when the coupling between the pointer and the system is much less than the pointer width. As reported in the literature, "the crux of [the] method is that the first measurement is performed in a gentle way through weak measurement, so as not to invalidate the second" [4] or "directly measuring [...] relies on the technique of weak measurement: extracting so little information from a single measurement that the state does not collapse" [8].

The interest about DWT is that the scheme in some cases may have experimental advantages over QST, in terms of simplicity, versatility, and directness [11]: it requires only a weak coupling of the system with an external pointer, a postselection of the final state of the system, and a simple projective measurement of two complementary observables of the pointer, a two-level system. QST, in contrast, requires measuring a complete set of noncommuting observables of the system, which can be a very demanding requirement in systems with a large number of degrees of freedom. For instance, the determination of the transverse spatial wave function of a single photon was first realized by DWT [4], as well as the measurement of a one-million-dimensional photonic state [16].

Here we show that the quantum wave function can be obtained by the same scheme used in DWT but using only strong measurements: with this term we here refer to measurements characterized by a strong coupling between the system and the pointer. As explained below, a strong measurement does not always coincide with a projective measurement on the system.

We thus demonstrate that the weak measurement is not necessary for the direct measurement of the wave function. We then compare DWT with our method, showing that the use of strong measurements in most cases gives a better estimation of the quantum wave function, outperforming DWT when both accuracy and precision are considered. Our analysis also allows one to evaluate how the wave function estimated by DWT is related to the correct wave function; see Eq. (5). We also solved an unresolved 
question related to DWT: how "weak" the interaction should be such that DWT gives a correct estimation of the wave function. In particular, we will derive a sufficient criterium for the applicability of DWT based on the measured probabilities; see Eq. (8).

Review of direct weak tomography. - Let us consider a $d$-dimensional Hilbert space with basis $\{|x\rangle\}$ with $x=1, \ldots, d$. The states $|x\rangle$ are equivalent to position eigenstates of a discretized segment. A generic pure state in this basis can be written as

$$
|\psi\rangle_{X}=\sum_{x=1}^{d} \psi_{x}|x\rangle .
$$

The scheme used in DWT is shown in Fig. 1: first, the following initial state $\left|\Psi_{\text {in }}\right\rangle=|\psi\rangle_{X} \otimes|0\rangle_{\mathcal{P}}$ is prepared, with $|0\rangle_{\mathcal{P}}$ the pointer state. The pointer belongs to a bidimensional qubit space spanned by the states $\left\{|0\rangle_{\mathcal{P}},|1\rangle_{\mathcal{P}}\right\}$ [17]. The system is then evolved according to the following unitary operator:

$$
U_{x}(\theta)=e^{-i \theta \hat{\pi}_{x} \otimes \hat{\sigma}_{y}} \approx \mathbb{1}-i \theta \hat{\pi}_{x} \otimes \hat{\sigma}_{y},
$$

where $\theta$ is an arbitrary angle and $\hat{\pi}_{x}=|x\rangle\langle x|$. The approximation of the right-hand side of Eq. (2) is obtained for small $\theta$. The previous evolution corresponds to a pointer rotation conditioned to $|\psi\rangle_{X}$ being in the state $|x\rangle$. A projective measurement on the pointer, weakly coupled to the photon position and followed by a projective measurement of the photon momentum, allows one to directly determine the wave function. Indeed, by postselecting only the outcomes corresponding to the zero transverse momentum state $\left|p_{0}\right\rangle=(1 / \sqrt{d}) \sum_{x}|x\rangle$, the (unnormalized) pointer state becomes $|\varphi\rangle_{\mathcal{P}} \approx(1 / \sqrt{d})\left[\tilde{\psi}|0\rangle_{\mathcal{P}}+\theta \psi_{x}|1\rangle_{\mathcal{P}}\right]$ with $\tilde{\psi}=\sum_{x=1}^{d} \psi_{x}$. The choice of $\left|p_{0}\right\rangle$ is arbitrary, and a different value of the transverse momentum might be needed for particular states, as explained below. Since a global phase is not observable, it is possible to arbitrarily choose the phase of $\tilde{\psi}$ : we set the latter phase such that $\tilde{\psi}$ is real valued and positive. In the first order in $\theta$, the wave function can be derived directly as [4]

$$
\psi_{W, x}=\frac{d}{2 \theta \tilde{\psi}}\left[\left(P_{+}^{(x)}-P_{-}^{(x)}\right)+i\left(P_{L}^{(x)}-P_{R}^{(x)}\right)\right],
$$

where $P_{j}^{(x)}$ represent the probabilities of measuring the pointer state into the diagonal basis $| \pm\rangle_{\mathcal{P}}=\frac{1}{\sqrt{2}}(|0\rangle \pm|1\rangle)$

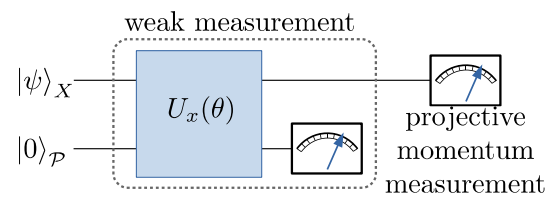

FIG. 1. Scheme of the original DWT method used to measure the wave function. or the circular basis $|L\rangle_{\mathcal{P}}=\frac{1}{\sqrt{2}}(|0\rangle+i|1\rangle)$ and $|R\rangle_{\mathcal{P}}=$ $\frac{1}{\sqrt{2}}(|0\rangle-i|1\rangle)$. We note that, since the (real positive) proportionality constant $(d / 2 \theta \tilde{\psi})$ is $x$ independent, it can be obtained at the end of the procedure by normalizing the wave function. The different probabilities can be also expressed in the framework of a positive operator-valued measure (POVM), as detailed in Supplemental Material [18]. From now on, we indicate with $\psi_{W, x}$ the (approximate) wave function obtained with the DWT method. We also define $\tilde{\psi}_{W} \equiv \sum_{x} \psi_{W, x}=(d / 2 \theta \tilde{\psi}) \sum_{x}\left(P_{+}^{(x)}-P_{-}^{(x)}\right)$, and we fix the global phase of $\psi_{W, x}$ by Eq. (3).

Relation (3) was generalized to mixed states in Ref. [11]. By repeating the measurements and changing the $x$ parameter in the evolution $U_{x}(\theta)$, the full wave function can be reconstructed. We now show that a relation similar to (3) can be obtained by strong or arbitrary strength measurements.

Arbitrary strength measurement.-Measurement with arbitrary strength is obtained by choosing an arbitrary value of $\theta$ within $0<\theta \leq \pi / 2$. We start our analysis with strong measurements, corresponding to $\theta=\pi / 2$. In this case the unitary operator (2) becomes $U_{x}(\pi / 2)=$ $\mathbb{1}-|x\rangle\langle x| \otimes\left(\mathbb{1}_{\pi}+i \sigma_{y}\right)$. After the interaction, the initial state $\left|\Psi_{\text {in }}\right\rangle$ is measured on the state $\left|p_{0}\right\rangle \otimes\left|\phi_{f}\right\rangle$, where $\left|\phi_{f}\right\rangle$ is the final polarization state. The amplitude for that transition is just $\mathcal{A}=\left\langle p_{0} \mid \psi\right\rangle_{X}\left\langle\phi_{f} \mid 0\right\rangle_{\mathcal{P}}-\psi_{x} \sqrt{2 / d}\left\langle\phi_{f} \mid-\right\rangle_{\mathcal{P}}$. This amplitude involves both the real and imaginary parts of $\psi_{x}$, so its magnitude squared does, too: by choosing different values of $\left|\psi_{f}\right\rangle$, it is possible to determine the real and imaginary parts of $\psi_{x}$. In particular, by choosing the final state $\left|\phi_{f}\right\rangle$ as $|1\rangle_{\mathcal{P}},|+\rangle_{\mathcal{P}},|-\rangle_{\mathcal{P}},|L\rangle_{\mathcal{P}}$, and $|R\rangle_{\mathcal{P}}$ states, the wave function can be obtained as

$$
\psi_{x}=\frac{d}{2 \tilde{\psi}}\left[\left(P_{+}^{(x)}-P_{-}^{(x)}+2 P_{1}^{(x)}\right)+i\left(P_{L}^{(x)}-P_{R}^{(x)}\right)\right] .
$$

To obtain the above relation, we fixed again $\tilde{\psi}=|\tilde{\psi}|$. It is very important to stress that, differently from the DWT method, the above result is exact, without any approximation. We denote the previous relations as the direct-strongtomography (DST) method. The difference with respect to the DWT is the need of measuring the pointer state also in the state $|1\rangle_{\mathcal{P}}$. This extra requirement is compensated by the fact that the result is not approximated and the accuracy and precision of the method overcome the DWT, as we will show in the following. We underline that the measurement in the $|1\rangle_{\mathcal{P}}$ state, and only in this state, corresponds to a projective measurement of the photon position, as the outcome of the measurement is proportional to $\left|\psi_{x}\right|^{2}$ (see [18]). On the contrary, a projection of the pointer in the $\{|+\rangle,|-\rangle\}$ or $\{|L\rangle,|R\rangle\}$ bases acts as a partial quantum erasure on the which-position information; therefore, a subsequent momentum postselection allows one to extract information about the real and imaginary parts of $\psi_{x}$. As 
detailed in Supplemental Material [18], for arbitrary $\theta$, the wave function can be obtained as $\operatorname{Re}\left(\psi_{x}\right) \propto P_{+}^{(x)}-P_{-}^{(x)}+$ $2 \tan (\theta / 2) P_{1}^{(x)}$ and $\operatorname{Im}\left(\psi_{x}\right) \propto P_{L}^{(x)}-P_{R}^{(x)}$.

Accuracy of DWT.-In the case of DWT, the obtained wave function $\psi_{W, x}$ is an approximation of the correct wave function $\psi_{x}$. We now evaluate the accuracy of the DWT, namely, the errors arising by using Eq. (3) in place of the exact values of (4). As done in Ref. [13], we define the accuracy in terms of the trace distance $\mathcal{D}$ between the correct wave function $\psi_{x}$ and the weak-value approximation $\psi_{W, x}$ [19], that for pure states reduces to $\mathcal{D}=\sqrt{1-\left|\left\langle\psi \mid \psi_{W}\right\rangle\right|^{2}}$. We first give the analytical expression of $\mathcal{D}$ in terms of the original wave function and then show how $\mathcal{D}$ can be upper bounded by using the measurement outcomes.

As shown in Supplemental Material [18], the relation between the exact wave function $\psi_{x}$ and the weak-value estimate $\psi_{W, x}$ given in (3) can be expressed by the following relation:

$$
\psi_{W, x}=\psi_{x} \frac{\tilde{\psi}-\epsilon_{\theta} \psi_{x}^{*}}{\mathcal{N}},
$$

with $\epsilon_{\theta} \equiv 2 \sin ^{2}(\theta / 2), \mathcal{N} \equiv \sqrt{\left|\tilde{\psi}-\epsilon_{\theta}\left\langle\psi_{x}\right\rangle\right|^{2}+\epsilon_{\theta}^{2} \sigma_{\psi}^{2}}$, and $\sigma_{\psi}^{2} \equiv\left\langle\left|\psi_{x}\right|^{2}\right\rangle-\left|\left\langle\psi_{x}\right\rangle\right|^{2}$. In the previous equation, $\sigma_{\psi}^{2}$ is the "variance" the wave function where the average is defined with respect to the probability density $p_{x}=\left|\psi_{x}\right|^{2}$, namely, $\left\langle\left|\psi_{x}\right|^{2}\right\rangle=\sum_{x}\left|\psi_{x}\right|^{4}$ and $\left\langle\psi_{x}\right\rangle=\sum_{x} \psi_{x}\left|\psi_{x}\right|^{2}$. By inserting (5) into the trace distance $\mathcal{D}$, we obtain

$$
\mathcal{D}=\frac{\epsilon_{\theta} \sigma_{\psi}}{\mathcal{N}}
$$

expressing $\mathcal{D}$ in terms of the original wave function $\psi_{x}$ and the interaction parameter $\theta$. The previous expression indicates when the weak measurement method can be efficiently used; indeed, when

$$
\mathcal{D} \ll 1,
$$

the approximate wave function $\psi_{W, x}$ correctly estimates the wave function $\psi_{x}$. Since Eq. (6) can be inverted into $\epsilon_{\theta} \sigma_{\psi}=\left(\mathcal{D} / \sqrt{1-D^{2}}\right)\left|\tilde{\psi}-\epsilon_{\theta}\left\langle\psi_{x}\right\rangle\right|$, for a small $\mathcal{D}$ condition (7) is equivalent to $\left(\epsilon_{\theta} \sigma_{\psi} /\left|\tilde{\psi}-\epsilon_{\theta}\left\langle\psi_{x}\right\rangle\right|\right) \ll 1 \quad$ (see Supplemental Material [18] for the detailed calculation).

Condition (7), however, cannot be used if the exact wave function $\psi_{x}$ is unknown. For this reason, we now present a sufficient condition for the application of the DWT method that is expressed in terms of the measured probabilities. As shown in Supplemental Material [18], when the following inequality is satisfied:

$$
\sum_{x}\left(P_{+}^{(x)}-P_{-}^{(x)}\right) \geq 0
$$

the systematic error is bounded by $\mathcal{D} \leq \theta / 2$ (for small $\theta$ ). We note that Eq. (8) is equivalent to $\tilde{\psi}_{W} \geq 0$ when the global phase of $\psi_{W, x}$ is fixed by Eq. (3).

If condition (8) is not satisfied, the DWT method is not guaranteed to work, and a lower $\theta$ should be chosen to achieve condition (8). Since $\tilde{\psi}_{W}$ can be expressed in term of the original wave function as $\tilde{\psi}_{W}=\left(\tilde{\psi}^{2}-\epsilon_{\theta} / \mathcal{N}\right)$, for any wave function with $\tilde{\psi} \neq 0$ it is possible to lower $\theta$ such that condition (8) is satisfied. The wave functions with $\tilde{\psi}=0$ corresponds to the set of "pathological" wave functions for which the DWT and the DST methods do not work for any values of $\theta$ if the momentum postselected state is $\left|p_{0}\right\rangle$. Indeed, if $\tilde{\psi}=0$, the systematic error (6) can be easily evaluated to be $\mathcal{D}=\sigma_{\psi} / \sqrt{\left\langle\left|\psi_{x}\right|^{2}\right\rangle}$ that is independent of $\theta$ : by changing the interaction parameter, the error cannot be lowered for such wave functions [20]. Also for DST, the proportionality constant $(d / 2 \tilde{\psi} \sin \theta)$ in (4) diverges if $\tilde{\psi}=0$. In such a case, a different momentum state for postselection different from $\left|p_{0}\right\rangle$ must be used.

To better evaluate the accuracy of the DWT, we have randomly chosen $10^{6}$ wave functions in a $d=10$ dimensional Hilbert space according to the Haar measure. We calculated for different values of $\theta$ the probability $p_{W}$ to violate the sufficient condition, namely, $p_{W}=$ $\operatorname{Prob}\left(\tilde{\psi}_{W}<0\right)$. We also calculated the probability $p_{\mathcal{D}}$ of having an error $\mathcal{D}$, evaluated by (6), larger than 0.1 . In Fig. 2, we show the probabilities $p_{W}$ and $p_{\mathcal{D}}$ in a function of $\theta$. In the inset, we also show the systematic error $\mathcal{D}$ in a function of $\sigma_{\psi} / \tilde{\psi}$ for different values of $\theta$. Since the distribution of $\mathcal{N}$ is peaked around $\tilde{\psi}$ for $\theta \leq 0.5$, it is possible to approximate $\mathcal{D} \approx \epsilon_{\theta}\left(\sigma_{\psi} / \tilde{\psi}\right)$ : indeed, the dashed lines in the inset in Fig. 2 represent the curves $\mathcal{D}=\epsilon_{\theta}\left(\sigma_{\psi} / \tilde{\psi}\right)$. The figure shows that, for low values of $\theta$, the DWT method fails with low probability and the systematic error is limited. Indeed, if we choose $\theta \leq 0.2$ for the $d=10$ case, we have $p_{W} \leq 1.75 \%$ and $p_{\mathcal{D}} \leq 0.57 \%$. Then, as expected, low values of the interaction parameter $\theta$ are suitable for the correct application of the DWT method. However, as we will show in the following, such low $\theta$ values lead to a larger statistical error

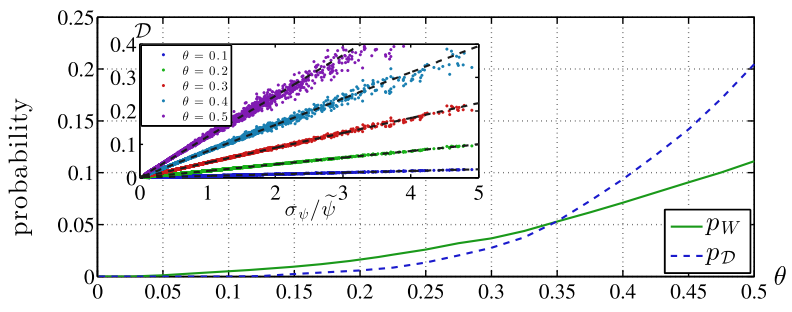

FIG. 2. Accuracy of the DWT: we show the probability $p_{W}$ of having $\tilde{\psi}_{W}<0$ and the probability $p_{\mathcal{D}}$ of having an error $\mathcal{D}$ larger than 0.1 . The inset shows trace distance $\mathcal{D}$ in a function of $\sigma_{\psi} / \tilde{\psi}$ for different values of $\theta$. We randomly choose $10^{6}$ wave functions in a $d=10$-dimensional Hilbert space. Dashed lines in the inset represent the curves $D=\epsilon_{\theta}\left(\sigma_{\psi} / \tilde{\psi}\right)$. 


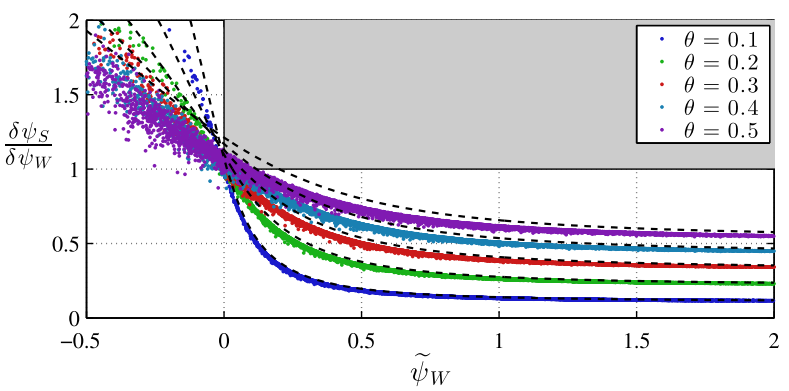

FIG. 3. Ratio of statistical errors $\left(\delta \psi_{S} / \delta \psi_{W}\right)$ in a function of $\tilde{\psi}_{W}$. The shaded area represents the points in which the DWT is convenient with respect to the DST method, corresponding to $\tilde{\psi}_{W} \geq 0$ and $\delta \psi_{W} \leq \delta \psi_{S}$.

(i.e., lower precision) compared to the strong measurement method.

Precision of the DWT.-An important performance parameter is the precision of the method, namely, the statistical errors on the estimated wave function. In particular, it is important to evaluate the scaling of such errors with the number of measurements. To this purpose, we evaluated the mean square statistical error $\delta \psi$ of the DWT and DST methods, obtained by summing the squares of the statistical error on the different $\psi_{x}$ :

$$
\delta \psi=\sqrt{\sum_{x}\left|\delta \psi_{x}\right|^{2}}
$$

As shown in Supplemental Material [18], the ratio between the statistical errors $\delta \psi_{S}$ and $\delta \psi_{W}$, respectively corresponding to the strong and weak method, can be approximately bounded by

$\frac{\delta \psi_{S}}{\delta \psi_{W}} \lesssim \sin \theta_{0} \sqrt{\frac{3}{2}} \sqrt{\frac{(2 d-5) \tilde{\psi}^{2}+2 \tilde{\psi}+8-2 / d}{(2 d-1) \tilde{\psi}^{2}+2 \epsilon_{\theta}\left(1-\tilde{\psi}-2 \tilde{\psi}^{2}\right)}}$,

where $\theta_{0}$ is the interaction parameter used for the weak measurement. The terms $\sin \theta_{0}$ in Eq. (10) shows that low values of $\theta_{0}$ correspond to a lower precision (i.e., larger statistical errors) of the DWT with respect to the DST method. In the statistical analysis, we compared the two methods by fixing the number of repetitions $N$ of the experiment; in the DWT or DST method, $N / 2$ or $N / 3$ repetitions are used for each basis, respectively. This is the origin of the $\sqrt{3 / 2}$ factor in Eq. (10).

For a complete demonstration of such a feature, we calculated the exact ratio $\left(\delta \psi_{S} / \delta \psi_{W}\right)$ for $10^{6}$ randomly chosen wave functions and compared it with the success parameter $\tilde{\psi}_{W}$ and the systematic error $\mathcal{D}$. The results are shown in Figs. 3 and 4. Figure 3 shows that, when the sufficiency condition for applying the DWT is satisfied (i.e., $\tilde{\psi}_{W} \geq 0$ ), the statistical errors of the DWT are typically greater than the errors of the DST. An approximate trend of the ratio $\delta \psi_{S} / \delta \psi_{W}$ can be obtained by noticing that,

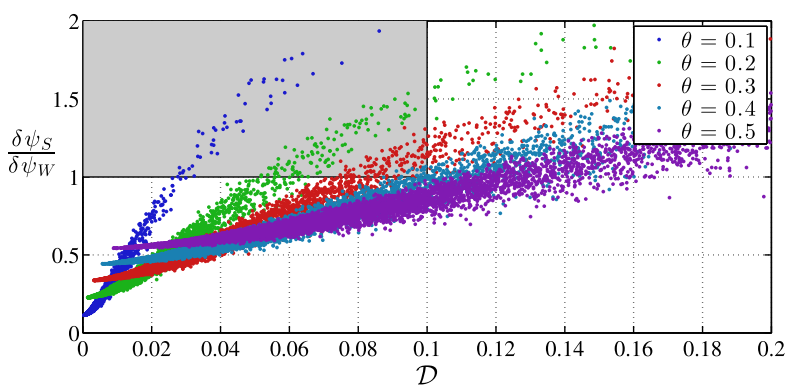

FIG. 4. Ratio of statistical errors $\left(\delta \psi_{S} / \delta \psi_{W}\right)$ in a function of $\mathcal{D}$. The shaded area represents the wave functions for which the statistical error of the DWT is lower than the DST method.

since $\mathcal{N} \approx \tilde{\psi}$, we can approximate $\tilde{\psi}_{W} \approx \tilde{\psi}-\epsilon_{\theta} / \tilde{\psi}$. Dashed curves in Fig. 3 represent the right-hand side of Eq. (10), with $\tilde{\psi}$ replaced by $\frac{1}{2}\left(\tilde{\psi}_{W}+\sqrt{\tilde{\psi}_{W}^{2}+4 \epsilon_{\theta}}\right)$, and well reproduce the behavior of the ratio $\delta \psi_{S} / \delta \psi_{W}$.

To further prove that the DST precision is typically greater than the DWT one, we plot in Fig. 4 the same ratio $\delta \psi_{S} / \delta \psi_{W}$ in a function of the exact trace distance $\mathcal{D}$ : for low systematic error $\mathcal{D}$, the statistical errors of the DWT are typically greater than the errors of DST. Equivalently, statistical errors of the DWT are reduced only as the systematic errors increase. Figure 4 shows that the DST precision overcomes the DWT one in most of the cases in which the DWT is accurate.

To better appreciate the above results, we plot in Fig. 5 the mean values of $\left(\delta \psi_{S} / \delta \psi_{W}\right)$ and $\mathcal{D}$ averaged over $10^{6}$ random wave functions in a function of $\theta$. The plot in Fig. 5 shows again that in order to lower the trace distance $\mathcal{D}$ it is necessary to decrease $\theta$. However, decreasing $\theta$, the statistical error $\delta \psi_{W}$ becomes larger than $\delta \psi_{S}$.

Mixed states. - The DWT can be generalized to determine the density matrix $\rho$ of mixed states, as shown in Ref. [11]. To directly measure $\rho$, the same method described for a pure state can be used, with the extra requirement that the strong measurement on momentum should be performed in all the momentum states $|p\rangle=(1 / \sqrt{d}) \sum_{x} e^{2 \pi i(p x / d)}|x\rangle$, while the pointer is measured in the $| \pm\rangle_{\mathcal{P}},|R\rangle_{\mathcal{P}},|L\rangle_{\mathcal{P}}$ states (as done for the pure state $\left.|\psi\rangle_{X}\right)$. We indicate by $\rho^{W}$ the density matrix that is reconstructed by the DWT and that approximates the correct matrix $\rho$. As shown in Supplemental Material [18], it can be expressed as

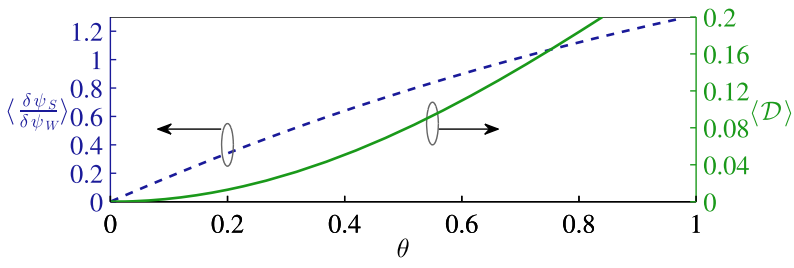

FIG. 5. Mean values of $\left(\delta \psi_{S} / \delta \psi_{W}\right)$ and $\mathcal{D}$ averaged over $10^{6}$ random wave functions in a function of $\theta$. 


$$
\rho^{W}=\frac{1}{\cos \theta}[\rho+(\cos \theta-1) D]
$$

with $D$ a diagonal matrix whose element are equal to the diagonal of $\rho$, namely, $D_{x, y}=\delta_{x, y} \rho_{x, x}$. By evaluating the accuracy of the DWT in terms of the trace distance $\mathcal{D}$ between $\rho$ and $\rho^{W}$, we obtained

$$
\mathcal{D}=\frac{1-\cos \theta}{2 \cos \theta} \operatorname{Tr}\left[\sqrt{(\rho-D)^{2}}\right] .
$$

Also in this case, the larger $\theta$ is, the larger $\mathcal{D}$ is and the lower the accuracy is in the estimation of $\rho$ by the DWT. Similarly to what we have shown for pure states, by performing an extra measurement of the pointer in the $|1\rangle_{\mathcal{P}}$ state, the exact expression of the density matrix can be obtained for any value of $\theta$ also in the case of mixed states (see Supplemental Material [18]).

Conclusions.-We have demonstrated that, in order to achieve a direct measurement of the wave function, weak measurements are not necessary. Indeed, we have shown that by using strong measurements, in which a large entanglement is achieved between the system and the pointer, a better estimation of the wave function, in terms of precision and accuracy, can be obtained for random matrices in most cases. Our method allowed us to derive a sufficient condition for the applicability of direct weak tomography. We believe that our results give a deeper understanding of the meaning of the weak value for the estimation of the wave function.

We thank P. Villoresi of the University of Padova and L. Maccone of the University of Pavia for useful discussions. Our work was supported by the Progetto di Ateneo PRAT 2013 (CPDA138592) of the University of Padova. G. V. also acknowledges the Strategic-Research-Project QUINTET of the Department of Information Engineering, University of Padova.

[1] D. F. V. James, P. G. Kwiat, W. J. Munro, and A. G. White, Phys. Rev. A 64, 052312 (2001).
[2] R. T. Thew, K. Nemoto, A. G. White, and W. J. Munro, Phys. Rev. A 66, 012303 (2002).

[3] A. I. Lvovsky and M. G. Raymer, Rev. Mod. Phys. 81, 299 (2009).

[4] J. S. Lundeen, B. Sutherland, A. Patel, C. Stewart, and C. Bamber, Nature (London) 474, 188 (2011).

[5] Y. Aharonov, D. Z. Albert, and L. Vaidman, Phys. Rev. Lett. 60, 1351 (1988).

[6] Y. Aharonov and L. Vaidman, Phys. Rev. A 41, 11 (1990).

[7] J. Dressel, M. Malik, F. M. Miatto, A. N. Jordan, and R. W. Boyd, Rev. Mod. Phys. 86, 307 (2014).

[8] J. Z. Salvail, M. Agnew, A. S. Johnson, E. Bolduc, J. Leach, and R. W. Boyd, Nat. Photonics 7, 316 (2013).

[9] M. Malik, M. Mirhosseini, M. P. J. Lavery, J. Leach, M. J. Padgett, and R. W. Boyd, Nat. Commun. 5, 3115 (2014).

[10] S. Kocsis, B. Braverman, S. Ravets, M. J. Stevens, R. P. Mirin, L. K. Shalm, and A. M. Steinberg, Science 332, 1170 (2011).

[11] J. S. Lundeen and C. Bamber, Phys. Rev. Lett. 108, 070402 (2012).

[12] J. Fischbach and M. Freyberger, Phys. Rev. A 86, 052110 (2012).

[13] L. Maccone and C. C. Rusconi, Phys. Rev. A 89, 022122 (2014).

[14] D. Das and Arvind, Phys. Rev. A 89, 062121 (2014).

[15] We used the term direct measurement to identify the method proposed [4]. As illustrated in Supplemental Material [18], the method can be described in the more general framework of POVMs.

[16] Z. Shi, M. Mirhosseini, J. Margiewicz, M. Malik, F. Rivera, and R. W. Boyd, arXiv:1503.04713.

[17] In the case of a photon spatial wave function, the pointer can be represented by a different degree of freedom of the photon, such as the polarization.

[18] See Supplemental Material at http://link.aps.org/ supplemental/10.1103/PhysRevLett.116.040502 for the detailed demonstrations of the results shown in the main text.

[19] We here recall that the trace distance between two quantum states $\rho$ and $\rho^{\prime}$ is defined as $\mathcal{D} \equiv \frac{1}{2} \operatorname{Tr}\left[\left|\rho-\rho^{\prime}\right|\right]$.

[20] For example, by using the DWT method on the following wave function $\psi_{1}=1 / \sqrt{2}, \psi_{2}=-1 / \sqrt{2}$, and $\psi_{x}=0$ for $x>2$ the statistical error is maximal, $\mathcal{D}=1$, for any value of $\theta$. 